\title{
A Stochastic Single-vendor Single-buyer Model under a Consignment Agreement
}

\author{
Ou Tang ${ }^{1}$, Simone Zanoni ${ }^{2}$ and Lucio Zavanella ${ }^{2}$ \\ ${ }^{1}$ Department of Management and Engineering, \\ Linköping Institute of Technology, \\ SE-581 83 Linköping, Sweden \\ 2 Dipartimento di Ingegneria Meccanica e Industriale, \\ Università degli Studi di Brescia, \\ Via Branze, 38, 25123 Brescia, Italy
}

\begin{abstract}
.
In the recent years, companies have begun to strengthen their supply agreements, such as sharing the management of inventories. This type of cooperation implies that the members of the supply chain share information and arrange a mutual agreement on their performance targets. The increased interest on supply chain topics has attracted researchers' attention to the problem of co-operation between the buyer and vendor, the two actors directly interacting in the supply mechanism. The present research investigates the way how a particular VMI policy, known as Consignment Stock (CS), may lead to a successful strategy for both buyer and vendor. The previous study [1] developed an analytical model of the CS policy, with reference to the centralised decision and deterministic settings. In order to fully explore the potentiality of CS policy, an extension of the model is proposed in this paper. The results indicate that the CS policy could be a strategic and profitable approach to improve supply chain performance in uncertain environments.
\end{abstract}

\section{Introduction}

Firms are no longer competitive as independent entities such as buyer and vendor, but rather as an integral part of supply chain. Thus the success of a firm depends on its managerial ability to integrate and coordinate the network of business relationships among the supply chain members. According to this scenario, Vendor Managed Inventory (VMI) represents an interesting approach to stock monitoring and control, progressively considered and introduced in both service and manufacturing industries. One VMI policy, known as Consignment Stock (CS), may suppress the vendor's inventory, as this actor will use buyer's warehouse to stock its 
finished products. Furthermore, the vendor guarantees that the quantity stored in the buyer's warehouse will be kept between a maximum and a minimum level, and thus further reduces the costs eventually induced by stock-out conditions. The buyer picks up the quantity of material needed to meet his production plans and, consequently, the material used is paid to the buyer.

The present study focuses on those situations where the deterministic conditions, necessary so as to apply Hill's model [2], do not prevail. The major effect determined by uncertain conditions is represented by stockout events (i.e., buyer's inability to promptly satisfy demand). This problem is generally approached by increasing reorder points and keeping safety stocks. Of course, an increased safety stock leads to the downstream movement of inventories, similarly to the CS approach [3]. In addition, in this study, we extend CS to a decentralised decision system in order to investigate the motivations and incentives for applying this policy.

A brief literature review is presented to summarise early studies in CS principle and relevant issues. The first study dealing with the integrated single-supplier singlecustomer problem is the paper by Goyal [4]. In [5] Banerjee considered the vendor manufacturing for stock at a finite rate and delivering the whole batch to the buyer as a single shipment. Goyal in [6] showed how lower-cost policies result from production batch splitting and multiple shipment delivery. Lu in [7] sets out the optimal production and equal-size shipment policy. Goyal in [8] demonstrated that lower cost policies may be obtained when shipments increase in size by a given ratio. Hill [9] derived the form of the optimal policy if shipment sizes may vary. More recently, Corbett [10] examined the impact of incentive conflicts and information asymmetry on performance in a two-player decentralized supply chain, which follows a continuous review $(\mathrm{Q}, \mathrm{R})$ policy. Corbett found out that, in the absence of a central planner with full information, no party may induce a joint optimal behaviour of all agents without sacrificing his own profits. In [11] a CS industrial case is presented while its analytical approach is treated in [1], together with some performance evaluation. Following in [12] a full analytical solution to CS is proposed. In [13] there is a summary of the previous research on the single-vendor single-buyer integrated production-inventory problem, in the deterministic cases.

According to the topics outlined, Section 2 presents the notation and system description. Section 3 focuses on the model formulation in a decentralised system and Section 4 discusses the results obtained by numerical examples. Finally, conclusions are drawn in Section 5.

\section{System description}

A single-vendor single-buyer supply chain is studied with a stochastic demand. The notations adopted are given below:

$A_{y}$ : $\quad$ production setup cost, paid by the vendor, $\epsilon$

$A_{b}$ : order emission cost, paid by the buyer, $\epsilon$

$h_{v}: \quad$ out-of-pocket inventory holding costs of vendor, E/unit/year

$h_{b}$ : out-of-pocket inventory holding costs of buyer, $\epsilon$ /unit/year

$\alpha: \quad$ interest rate 
D: $\quad$ demand rate, units/year

$P$ : $\quad$ buyer's purchasing price of the product, $\epsilon /$ unit

$m$ : vendor's marginal cost of product, $\epsilon /$ unit

$Q: \quad$ transportation batch size, units

$n$ : number of transportations within one production batch

$b$ : $\quad$ backorder cost, $\epsilon$ /unit

$T C_{v}$ total costs of vendor, $\epsilon$

$T C_{b}$ total costs of buyer, $\epsilon$

$\sigma_{L}: \quad$ standard deviation of demand during lead time

$k$ : safety stock factor

$f\left(\mu_{\mathrm{v}}\right)$ : probability density function of a standard normal distribution and we also have

$$
\begin{aligned}
& F(k)=\int f\left(\mu_{0}\right) d \mu_{0} \\
& G(k)=\int\left(\mu_{0}-k\right) f\left(\mu_{v}\right) d \mu_{0}
\end{aligned}
$$

In this system, the vendor supplies products to a buyer facing a stochastic demand, which is assumed to be normally distributed. The lead time for transportation is constant and equal to $L$. The lead time for production at the vendor site is null (i.e., the vendor does not need safety stocks). The buyer uses a continuous review reorder point inventory control system. Thus, the buyer's decision variables are the order quantity $Q$ and the reorder point $s$ (further related to the safety factor $k$ ). Once given $Q$ and $s$, the vendor will consequently determine the number of transportations $n$.

The present study distinguishes between the capital tied-up and the out-of-pocket inventory holding cost. Conventionally, the total inventory holding cost is assumed to be increasing when items move downstream in a supply chain. According to our industrial experience, this statement may sometime fail. In particularly, the out-ofpocket inventory holding cost does not have to follow the above assumption, e.g. when a specialised producer with limited storage space supplies a large manufacturer with low-cost bulk storage facilities [13].

\section{Model formulation in a decentralised system}

The early literature about CS $[1,11,12]$ assumes that production and transportation decisions are centrally made. This assumption may not always be true (e.g., for multiple business entities with conflicting objectives within a supply chain). The present study investigates the motivation of using CS contract and evaluates the performance of a supply chain with a powerful buyer. Therefore, the buyer is assumed to impose his optimal decisions $Q$ and $k$, and the vendor consequently may decide his production batch size $(n \cdot Q)$. Finally, the expected on-hand inventory is considered equal to the expected net one (on-hand minus backorders), as a standard approximation in inventory modelling (e.g. [14] and [15]). 


\subsection{Without a consignment stock contract}

The following formulae propose the vendor and buyer total costs:

$$
\begin{aligned}
& T C_{r}=\frac{A_{r}}{n} \frac{D}{Q}+\left(\alpha m+h_{r}\right)\left(\frac{n Q}{2}\right)-\left(\alpha m+h_{r}\right)\left(\frac{Q}{2}+k \sigma_{l}\right) \\
& T C_{b}=A_{t} \frac{D}{Q}+\left(\alpha P+h_{b}\right)\left(\frac{Q}{2}+k \sigma_{L}\right)+\frac{D b \sigma_{l} G(k)}{Q}
\end{aligned}
$$

The buyer will minimize his cost by using optimal order quantity and safety factor. In case an optimal $Q$ has been used, Equation 2 can be rewritten as

$$
T C_{b}^{*}=\sqrt{2 D\left(A_{b}+b \sigma_{l} G(k)\right)\left(\alpha P+h_{b}\right)}+\left(\alpha P+h_{b}\right)\left(k \sigma_{l}\right)
$$

Formula (3) may be differentiated with respect to $k$ and set equal to zero

$$
\frac{\partial T C_{h}{ }^{*}}{\partial k}=-\frac{D\left(b \sigma_{l} F(k)\right)\left(\alpha P+h_{b}\right)}{\sqrt{2 D\left(A_{b}+b \sigma_{L} G(k)\right)\left(\alpha P+h_{b}\right)}}+\left(\alpha P+h_{b}\right)\left(\sigma_{L}\right)=0
$$

This ends with the optimisation conditions:

$$
\frac{A_{b} / b \sigma_{L}+G(k)}{F(k)^{2}}=\frac{D b}{2 \sigma_{L}\left(\alpha P+h_{b}\right)}
$$

and

$$
Q=\sqrt{\frac{2\left(A_{b} D+D b \sigma_{l} G(k)\right)}{\left(\alpha P+h_{b}\right)}}=\frac{D b}{\left(\alpha P+h_{b}\right)} F(k)
$$

Once the buyer's optimal safety factor and order quantity have been obtained, the optimal value of $n$ may be found from the difference equation of $T C_{v}$, i.e. the optimal $n$ should be the smallest integer satisfying

$$
n(n+1) \geq \frac{2 A_{v} D}{\left(\alpha m+h_{n}\right)} \frac{1}{Q^{2}}
$$

\subsection{A consignment contract}

A CS contract implies that the vendor pays the capital tied up cost of the stocks delivered to the buyer but not yet sold. Thus, the total costs change as follows

$$
\begin{aligned}
& T C_{r}{ }^{\prime}=\frac{A_{r}}{n} \frac{D}{Q}+\left(\alpha m+h_{r}\right)\left(\frac{n Q}{2}\right)-h_{r}\left(\frac{Q}{2}+k \sigma_{L}\right) \\
& T C_{b}{ }^{\prime}=A_{b} \frac{D}{Q}+h_{b}\left(\frac{Q}{2}+k \sigma_{L}\right)+\frac{D b \sigma_{L} G(k)}{Q}
\end{aligned}
$$

Using the same approach as in Section 3.1, the optimisation conditions for the buyer are:

$$
\frac{A_{b} / b \sigma_{L}+G(k)}{F(k)^{2}}=\frac{D b}{2 \sigma_{L} h_{b}} \text { and } Q=\frac{D b}{h_{b}} F(k)
$$


The corresponding optimal $n$ should follow:

$$
n(n+1) \geq \frac{2 A_{v} D}{\left(\alpha m+h_{v}\right)} \frac{1}{Q^{2}}
$$

\subsection{Comparison and analysis}

Proposition 1: $F(k)^{2} / G(k)$ is a decreasing function with respect to $k$, for $k>0$. This can be easily observed in the function curve given in Fig.1 below.

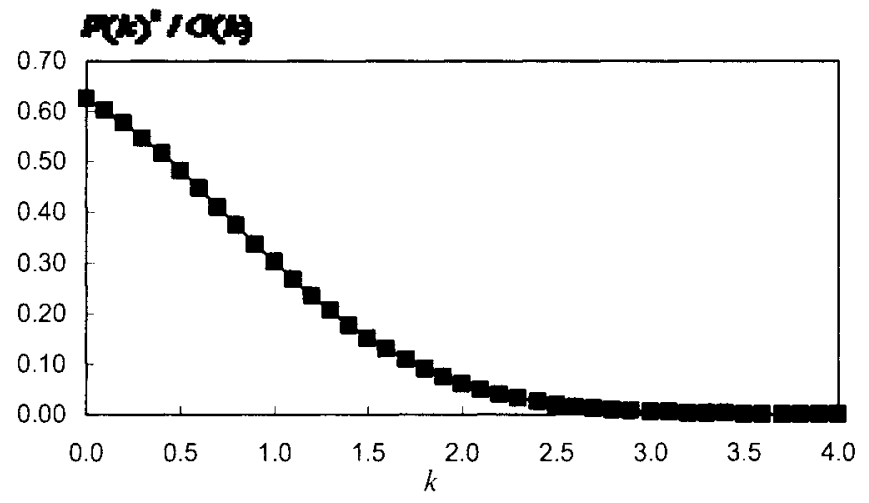

Fig. 1. $F(k)^{2} / G(k)$ as a function of $k$ for a standard normal distribution

Proposition 2: the service level, compared to a policy without CS, is improved by CS adoption.

Both $F(k)$ and $F(k)^{2} \cdot G(k)^{-1}$ are decreasing functions of $k$. Thus, $(A+G(k)) \cdot F(k)^{-2}$ is an increasing function with respect to $k$ ( $A$ is a positive constant). Since $\frac{D b}{2 \sigma_{L}\left(\alpha P+h_{b}\right)}<\frac{D b}{2 \sigma_{L} h_{b}}$, a larger optimal $k$ value is found for the CS case, as compared to the one without consignment. In addition, a higher safety factor will then lead to a higher service level.

Proposition 3: with a CS contract, the optimal order quantity shall follow the ratio

$$
\frac{Q^{*}}{Q^{*}}=\frac{\alpha P+h_{b}}{h_{b}} \frac{\left.F\left(k^{* *}\right)\right)}{\left.F\left(k^{*}\right)\right)}
$$

The change in the order quantity is a balance between the inventory holding cost ratio and the changes of $k$ value (correspondingly, the $F(k)$ value).

Proposition 4: The CS contract always reduces the buyer's cost.

Any $Q$ and $k$ values (including $Q^{*}$ and $k^{*}$ for $T C_{b}$ ) determine $T C_{b}>T C_{b}$ ', as easy to see comparing Equations 2 and 9. 
Proposition 5: Vendor's optimal cost can be reduced when

$$
\frac{\left(\alpha m+h_{v}\right)}{h_{r}}<\frac{\left(Q^{*}+2 k^{*} \sigma_{L}\right)}{\left(Q^{*}+2 k^{*} \sigma_{L}\right)}
$$

This result emerges from the following relationship:

$$
T C_{r}{ }^{*}{ }^{*}-T C_{r}{ }^{*}=\left(\alpha m+h_{r}\right)\left(\frac{Q^{*}}{2}+k^{*} \sigma_{l}\right)-h_{v}\left(\frac{Q^{\prime *}}{2}+k^{\prime *} \sigma_{l}\right)<0
$$

\section{Illustrative examples}

The set of data offered in the present examples are slightly changed from the case of an Italian company producing braking systems for the automotive industry. Focus is given on brake pads (an assembly component in the company manufacturing cycle). The specialised vendor of the brake pads is close to the company (for a detailed description of the case study see [1]). Parameters values are given below:

$$
\begin{aligned}
& A_{\nu}=400 € \text { and } A_{b}=70 \epsilon \\
& h_{v}=0.175 \epsilon / \text { unit/year } \text { and } h_{b}=0.15 \epsilon / \text { unit/year } \\
& \alpha=10 \% \\
& D=50000 \text { units/year } \\
& P=2.5 \epsilon / \text { unit } m=2 \epsilon / \text { unit } ; b=0.25 \epsilon / \text { unit } \\
& \sigma_{L}=300 \text { (Lead time } L \text { is about } 1 \text { week) }
\end{aligned}
$$

Let us consider the policy without CS. In the first step, the safety factor is evaluated by Equation $3 . F(k)$ and $G(k)$ values can be easily obtained by standard normal distribution table or, alternatively, using Waissi and Rossin approximation [15]. An upper bound $k$ can be used as an initial value for searching Equation 3 solution by examining

$$
\frac{A_{b} / b \sigma_{l}}{F(\bar{k})^{2}}=\frac{D b}{2 \sigma_{L}\left(\alpha P+h_{b}\right)}
$$

Since $F(k)$ is a decreasing function of $k$, and $G(k) \cdot F(k)^{-2}$ is positive, the optimal $k$ must be smaller than $\vec{k}$, as obtained by (12) (for the example, $\bar{k}=1.11$ and $k=1.09$ ). Inserting the results into Equation 6, optimal $Q=4309$ and Equation 7 provides the optimal $n=2$. The results are summarised in the left side of Table 1 below.

An additional case (Case 2) illustrates how the vendor's cost can be reduced by $\mathrm{CS}$ as well. Case 2 differs only in the marginal production cost, which is set to $m=0.5$. Results are given in the right part of Table 1 .

It can easily be seen that the service levels of the system are improved from $86.2 \%$ to $91.6 \%$. The buyer's cost is significantly reduced, both in terms of ordering, inventory holding and stockout costs. Vendor's costs may increase or decrease, depending on the production batch size and the changes in inventory holding cost (base case and Case 2, respectively) 
Table 1. Optimal decisions and costs

\begin{tabular}{|l|l|l|l|l|l|l|l|}
\hline \multicolumn{4}{|c|}{ Base case } & \multicolumn{5}{c|}{$m$} \\
\hline \multicolumn{2}{|c|}{ Without consignment } & \multicolumn{2}{|c|}{ With consignment } & \multicolumn{2}{c|}{ Without consignment } & \multicolumn{2}{c|}{ With consignment } \\
\hline$k$ & 1.09 & $k$ & 1.38 & $k$ & 1.09 & $k$ & 1.38 \\
\hline$Q$ & 4309 & $Q$ & 6983 & $Q$ & 4309 & $Q$ & 6983 \\
\hline$S L^{*}$ & 2 & $n$ & 2 & $n$ & 3 & $n$ & 2 \\
\hline$T C_{r}$ & $86.2 \%$ & $S L^{*}$ & $91.6 \%$ & $S L^{*}$ & $86.2 \%$ & $S L^{*}$ & $91.6 \%$ \\
\hline$T C_{h}$ & 3006 & $T C_{r}$ & 3369 & $T C_{r}$ & 2443 & $T C_{r}$ & 2320 \\
\hline$T C_{r}+T C_{h}$ & 4866 & $T C_{h}$ & 1108 & $T C_{h}$ & 1866 & $T C_{h}$ & 1108 \\
\hline & 4872 & $T C_{r}+T C_{h}$ & 4477 & $T C_{r}+T C_{h}$ & 4309 & $T C_{r}+T C_{h}$ & 3428 \\
\hline
\end{tabular}

$S L^{*}=$ service level $=1-F(k)$

\section{Conclusions}

This study presented a model to optimise the production and inventory decision in a single-vendor and single-buyer system with a stochastic demand. The optimisation models and the propositions of this paper can be applied to support a CS decision. The results show that, by using a CS principle and moving a part of the inventory holding cost to the vendor, the system performance can be improved, both in terms of total costs and service levels. Furthermore, a CS contract consistently reduces buyer's costs, but it may impact differently on the vendor's cost. Nevertheless, as already observed in the introduction, the vendor still perceives some advantages as a counterpart. For instance, he may manage his production plans more flexibly and the relationships between the vendor and the buyer can be improved. Moreover, a part of the channel costs reduction may be shared between the two actors and it can be used as an important element of the negotiation between the two actors of the chain. Finally, Sensitivity analysis and numerical experiments may allow an improved understanding of the circumstances determining the vendor benefits. Extending the model to positive and stochastic production lead time could be of great interest.

\section{References}

1. M. Braglia and L. Zavanella, Modelling an industrial strategy for inventory management in supply chains: the 'Consignment Stock' case, Int. Jour. of Prod. Res. 41, 3793-3808 (2003).

2. R.M. Hill, The single-vendor single-buyer integrated production-inventory model with a generalised policy, Europ. Jour. of Oper. Res. 97, pp.493-499 (1997).

3. L. Abdel-Malek; G. Valentini and L. Zavanella, Managing stocks in Supply Chains: modelling and issues, in Seuring S.; Goldbach M., Cost Management in Supply Chains, Heildeberg: Physica Verlag, 325-335 (2002).

4. S.K. Goyal, Determination of optimum production quantity for a two-stage production system, Oper. Res. Quart. 28, 865-870 (1977). 
5. A. Banerjee, A joint economic lot-size model for purchaser and vendor, Dec. Science 17, 292-311 (1986).

6. S.K. Goyal, A joint economic lot size model for purchaser and vendor: A comment. Dec. Science 19, $236-241$ (1988).

7. L. Lu, A one-vendor multi-buyer integrated inventory model. Europ. Jour. of Oper. Res. 82, 209-210 (1995).

8. S.K. Goyal and Y. P. Gupta, Integrated inventory models: the buyer-vendor coordination, Europ. Jour. of Oper. Res. 41, 261-269 (1989).

9. R.M. Hill, The optimal production and shipment policy for a single-vendor singlebuyer integrated production-inventory problem, Int. Jour. of Prod. Res. 37, 2463 2475 (1999).

10. C.J. Corbett, Stochastic inventory systems in a supply chain with asymmetric information: cycle stocks, safety stocks, and consignment stock, Oper. Res. 49(4), 487-500 (2001).

11. G. Valentini and L. Zavanella, The Consignment Stock of Inventories: Industrial Case and Performance Analysis, Int. Jour. of Prod. Econ., 81-82, 215-224 (2003).

12. S. Zanoni and R.W. Grubbstrom, A note on an industrial strategy for stock management in supply chains: modelling and performance evaluation, Int. Jour. of Prod. Res. 42, 4421-4426 (2004).

13. R.M. Hill and M. Omar, Another look at the single-vendor single-buyer integrated production-inventory problem, Int. Jour. of Prod. Res. 44(4), 791-800 (2006).

14. M. A. De Bodt and S. C. Graves, Continuous-review policies for a multiechelon inventory problem with stochastic demand, Manag. Science 31(10), 12861299 (1985).

15. E.A. Silver, D.F. Pyke and R. Peterson, Inventory Management and Production Planning and Scheduling, John Wiley and Sons, New York (1998). 